\title{
A quick evaluation method of nosocomial infection risk for cancer hospitals during the COVID-19 pandemic
}

\author{
Zaixiang Tang $^{1} \mathbb{D} \cdot$ Bei Sun $^{2} \cdot$ Bo Xu $^{2}$
}

Received: 8 April 2020 / Accepted: 23 April 2020 / Published online: 27 April 2020

c) Springer-Verlag GmbH Germany, part of Springer Nature 2020

\begin{abstract}
Nosocomial infections caused by COVID-19 will result in the immediate closure of the infected medical facility due to the highly contagious nature of COVID-19, further worsening medical shortages. We performed a Delphi consulting study to develop a risk model based on the experience of recently resumed activities in many cancer hospitals in China. Similar method had been used in a Chinese cancer hospital and effectively reduced the nosocomial Infection. We believe that the model can quickly identify COVID-19 cluster risks for medical facilities. The evaluation model could also be integrated into the hospital's emergency response system in a timely manner.
\end{abstract}

Initially an epidemic and now pandemic, the spread of COVID-19 has posed a significant challenge for medical responses around the world. Due to the large number of outbreaks in Asian, America, and European countries, much medical attention has been shifted to emergency care of large numbers of suspected or confirmed COVID-19 patients. As a result, timely treatment for patients with existing acute and chronic diseases such as cancer is on hold in many areas of the world. This is particularly devastating for cancer patients when delayed diagnosis and treatment will significantly affect long-term survival. It is estimated that tens of millions of cancer patients are under the threat of COVID-19 in the world (Bray et al. 2018). Human to human transmission of COVID-19 comes through respiratory droplets and close contact (Rothe et al. 2020). Cluster infections have occurred in families and shopping malls as well as in hospitals/clinics. Nosocomial infections, which affect both health care professionals and patients, will result in the immediate closure of the infected medical facility due to the highly contagious nature of COVID-19, further worsening medical shortages.

\footnotetext{
Zaixiang Tang

tangzx@suda.edu.cn

$\triangle$ Bo Xu

xubo@tmu.edu.cn

1 Department of Epidemiology and Biostatistics, School of Public Health, Medical College of Soochow University, Suzhou 215123, China

2 Tianjin Medical University Cancer Institute and Hospital, Tianjin 300060, China
}

Cancer patients have also been reported as having a higher risk of contracting COVID-19 (Liang et al. 2020), so posing a greater threat. Therefore, at this special time, there is an urgent need to categorize the cluster infection risk for health care facilities when cancer patients come to the clinic. To reach this goal, we performed a Delphi consulting study to develop a risk model based on the experience of recently resumed activities in many cancer hospitals in China. We first listed raw items that are relevant to the potential risk, based on three characteristics: epidemiological history, cancer-related factors or other concurrent medical related diseases. We then invited five clinical and public health experts to design a questionnaire based on this information, which we then sent to about 100 senior clinical experts in major cancer hospitals in China to rank the importance of each item for risk. We received 83 valid questionnaires that were analyzed for risk factor ranking (Fig. 1). High-risk factors include an epidemiological history, such as traveling to a COVID-19 affected region or having contact with confirmed or suspected COVID-19 persons. In addition, if the patient has cold symptoms, such as fever or cough, or a close companion meets the above criteria, the risk level is high for nosocomial infections. Intermediate risk factors include medical needs for oral/pulmonary function test and/or respiratory examinations, medical needs for invasive examinations, such as biopsies, endoscopy examinations, needs for thoracic or oral and maxillofacial surgery, and/or oncological emergencies. Patients who have self-quarantined for 2 weeks pose a low risk. Unlike the above-mentioned medical needs, patients with existing chronic conditions 
Fig. 1 A quick evaluation model of nosocomial infection risk

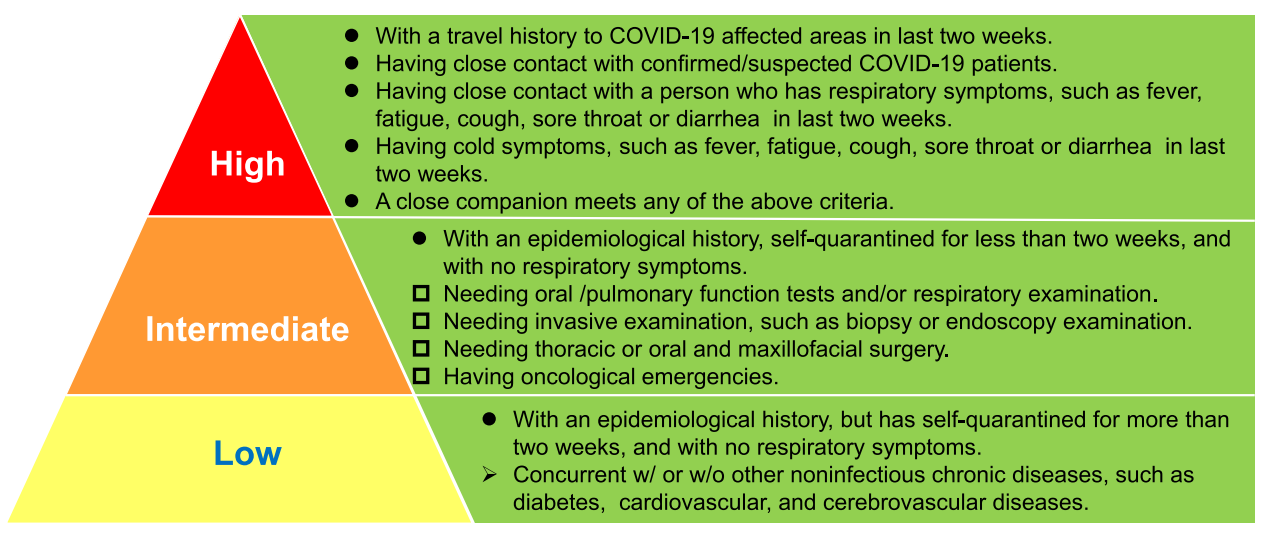

such as hypertension, diabetes, cardiovascular and/or cerebrovascular diseases pose a low risk. Similar methods have been used in many cancer hospitals in China and have effectively reduced nosocomial infections. We believe that the model could help identify COVID-19 cluster risks for medical facilities to implement specific plans to help these patients, and it can be tested globally.

Acknowledgements We thank more than 100 physicians from major cancer hospitals in China for their valuable comments on filling out the questionnaire.

\section{Compliance with ethical standards}

Conflict of interest The authors declare that they have no conflict of interests.

\section{Reference}

Bray F, Ferlay J, Soerjomataram I, Siegel RL, Torre LA, Jemal A (2018) Global cancer statistics 2018: GLOBOCAN estimates of incidence and mortality worldwide for 36 cancers in 185 countries. CA Cancer J Clin 68(6):394-424

Liang W, Guan W, Chen R et al (2020) Cancer patients in SARSCoV-2 infection: a nationwide analysis in China. Lancet Oncol 21(3):335-337

Rothe C, Schunk M, Sothmann P et al (2020) Transmission of 2019nCoV infection from an asymptomatic contact in Germany. N Engl J Med 382(10):970-971

Publisher's Note Springer Nature remains neutral with regard to jurisdictional claims in published maps and institutional affiliations. 